\section{Differential expression of mitogen activating protein kinases in periodontitis}

Travan S, Li F, D’Silva NJ, Slate EH, Kirkwood KL. Differential expression of mitogen activating protein kinases in periodontitis. J Clin Periodontol 2013; 40: 757-764. doi: 10.1111/jcpe.12123.

\begin{abstract}
Aim: Following toll-like receptor (TLR) engagement, lipopolysaccharide (LPS) can stimulate the expression of pro-inflammatory cytokines thus activating the innate immune response. The production of inflammatory cytokines results, in part, from the activation of kinase-induced signalling cascades and transcriptional factors. Of the four distinct classes of mitogen-activated protein kinases (MAPK) described in mammals, p38, c-Jun N-terminal activated kinases (JNK1-3) and extracellular activated kinases $($ ERK1,2) are the best studied. Previous data have established that p38 MAPK signalling is required for inflammation and bone loss in periodontal disease pre-clinical animal models.

Materials \& Methods: In this study, we obtained healthy and diseased periodontal tissues along with clinical parameters and microbiological parameters. Excised fixed tissues were immunostained with total and phospho-specific antibodies against p38, JNK and ERK kinases.

Results: Intensity scoring from immunostained tissues was correlated with clinical periodontal parameters. Rank correlations with clinical indices were statistically significantly positive $(p$-value $<0.05)$ for total p38 (correlations ranging 0.49 0.68), phospho-p38 (range 0.44-0.56), and total ERK (range 0.52-0.59) levels, and correlations with JNK levels also supported association (range 0.42-0.59). Phospho-JNK and phospho-ERK showed no significant positive correlation with clinical parameters of disease.

Conclusion: These data strongly implicate p38 MAPK as a major MAPK involved in human periodontal inflammation and severity.
\end{abstract}

\author{
Suncica Travan ${ }^{1}$, Fei $\mathbf{L i}^{1}$, \\ Nisha J. D'Silva ${ }^{1,5}$, Elizabeth $H$. \\ Slate $^{2,3}$ and Keith L. Kirkwood ${ }^{3,4}$ \\ ${ }^{1}$ Department of Periodontics and Oral \\ Medicine, School of Dentistry, University of \\ Michigan, Ann Arbor, MI, USA; ${ }^{2}$ Department \\ of Statistics, Florida State University, \\ Tallahassee, FL, USA; ${ }^{3}$ Department of \\ Craniofacial Biology and Center for Oral \\ Health Research, Medical University of South \\ Carolina, Charleston, SC, USA; ${ }^{4}$ Department \\ of Microbiology and Immunology, Medical \\ University of South Carolina, Charleston, SC, \\ USA; ${ }^{5}$ Department of Pathology, Medical \\ School, University of Michigan, Ann Arbor, \\ MI, USA
}

Key words: cell signalling; inflammation; mitogen activating protein kinases; periodontitis

Accepted for publication 4 May 2013
In the periodontal microenvironment, bacterial constituents including gramnegative derived lipopolysaccharide (LPS) can initiate inflammatory bone

\section{Conflict of interest and source of} funding statement

The authors have no conflict of interest to declare. NIH DE018290, DE021423, DE019513 and 2P20RR017696 supported funding for this study. loss as appreciated in periodontal diseases. LPS can stimulate the expression of IL- $1 \beta$, TNF- $\alpha$, IL- 6 and receptor activator of $\mathrm{NF} \kappa \mathrm{B}$ Ligand (RANKL) by activating the innate immune response as well as nonimmune cells such as gingival fibroblasts (Nakashima et al. 2000, Jiang et al. 2002, Kikuchi et al. 2003). The production of inflammatory cytokines results from the activation of kinase-induced signal- ling cascades and transcriptional factors. Within periodontal diseased tissues monocytes, macrophages and fibroblasts, all produce cytokines such as TNF- $\alpha$, IL- $1 \beta$, PGE $_{2}$ and IL-6 (Lee et al. 1995, Reddi et al. 1996) and have all been found to be significantly elevated in diseased periodontal sites compared to healthy or inactive sites (Stashenko et al. 1991, Geivelis et al. 1993, Gamonal et al. 2000, Ejeil et al. 2003, Gorska et al. 
2003). These cytokines orchestrate the cascade of destructive events that occur in the periodontal tissues, and trigger the production of an array of inflammatory enzymes and mediators including MMPs and prostaglandins. Moreover, pro-inflammatory cytokines directly or indirectly recruit and activate osteoclasts through RANKL-dependent and independent pathways, resulting in irreversible periodontal bone destruction (Assuma et al. 1998, Graves 1999). Therefore, knowledge regarding the molecular pathways that govern expression of many inflammatory mediators may have therapeutic significance in the management of inflammatory diseases including chronic periodontitis.

Bacterial LPS initiates the innate immune signalling cascade through interaction with cell surface protein expressed CD14 as well as toll-like receptors (TLRs), mainly TLR-2 and TLR-4 (Kirschning et al. 1998, Yang et al. 2000, Kirschning \& Bauer 2001). Expression of both TLR-2 and TLR-4 is increased in periodontal disease tissues, suggesting that these receptors have an increased capacity to signal and influence downstream cytokine expression (Mori et al. 2003, Beklen et al. 2008). TLR-4 signalling activates MyD88-dependent pathways to subsequent activation of interleukin1R-associated kinase (IRAK), tumour necrosis factor receptorassociated factor-6 (TRAF6) and ultimately nuclear factor kappa B $(\mathrm{NF}-\kappa \mathrm{B})$ required for cytokine induction. Also, TRAF6-dependent pathways are required for recruitment of different adaptor proteins and activation of various mitogen activating protein kinases (MAPK) and activation of nuclear factor kappa $\mathrm{B}(\mathrm{NF}-\kappa \mathrm{B})$. The transcription factors $\mathrm{NF}-\kappa \mathrm{B}$ and activator protein1 (AP-1) control expression of some of the most common inflammatory mediators present in periodontal inflammation, including IL-1, IL-6, TNF- $\alpha$ and MMPs [reviewed in (Kirkwood \& Rossa 2009)].

Although both MAPK pathways and $\mathrm{NF}-\kappa \mathrm{B}$ signalling pathways are well established in regulation of inflammatory cytokines and MMP genes in periodontal diseases, considerable less information is known about MAPK than $\mathrm{NF}-\kappa \mathrm{B}$ signalling in human disease. MAPKs phosphorylate a number of intracellular targets, including transcription factors (e.g. AP-1 and CHOP) and RNA-binding proteins (e.g. tristetraprolin and $\mathrm{HuR}$ ), which tightly regulate expression of inflammatory genes including at transcriptional and posttranscriptional levels. Target genes downstream of MAPK signalling include many common inflammatory mediators such as IL-1, IL-6, TNF- $\alpha$, COX-2 and MMPs [recently reviewed in (Palanisamy et al. 2012)].

Understanding the cell signalling processes involved in periodontal disease progression and destruction is key in the development of new therapeutic approaches to prevent or attenuate periodontal destruction. Pre-clinical periodontal disease models indicate that there is a strong positive correlation between activation of MAPKs, inflammation and bone loss (Kirkwood et al. 2007a, Rogers et al. 2007, Kirkwood \& Rossa 2009, Li et al. 2011, 2012). However, no data regarding the significance of MAPK activation in periodontal disease in humans have been evaluated. In the study, clinical periodontal parameters were correlated with the level of activation of MAPKs in subjects with chronic periodontal disease compared to healthy controls. Data obtained strongly indicate that total p38 and ERK have a significant positive correlation with periodontal disease severity while only phosphorylated p38 only correlates with clinical parameters of periodontal disease progression.

\section{Materials and Methods}

This study was approved by the Institutional Review Board for the Health Sciences at the University of Michigan, Ann Arbor, MI, USA. A total of twenty-one tissue samples from human subjects were included in the study (13 females and 8 males). The subjects were divided into two groups: healthy (10 subjects) and diseased (11 subjects). Informed consent was obtained with all patients prior to initiating study.

Prior to surgery, clinical parameters were measured at the same sites where tissues were harvested including: Plaque index (PI) on a scale of $0-3$ (0-no plaque, 1-w/probe, 2-visible, 3-abundant) (Loe 1967), Gingival Index (GI) on a scale of 0-3 (0-no inflammation, 1-mild, 2-moderate w/BOP, 3-severe, spontaneous) bleeding on probing (BOP), pocket depth (PD), BOP, gingival recession (REC) and clinical attachment level (CAL). Based on these parameters the inclusion criteria for the diseased group consisted of: at least 1 site with $\mathrm{PD}>4 \mathrm{~mm}$, GI 1-3 and PI 1-3. For the healthy controls acceptable parameters were $\mathrm{PD} \leq 4 \mathrm{~mm}, \mathrm{GI} \leq 1$ and $\mathrm{PI} \leq 2$. The exclusion criteria for both groups included: smokers, unstable systemic diseases or chronic disorders (diabetes, rheumatoid arthritis), patients using steroids, antibiotics, NSAIDS and/or other host modulators. The procured samples were from tissues that would have been otherwise discarded after periodontal surgery and or extraction sites. When clinically indicated, procured tissues included connective tissue near the sulcular epithelium.

\section{Microbial assessments: BANA test}

The benzoyl-DL-arginine-naphthylamide test was used to determine the presence of "red-complex" periodontal pathogens (Porphyromonas gingivalis, Treponema denticola and Tannerella forsythia) (Loesche et al. 1990) due to the unique ability of these pathogens to hydrolyse a synthetic trypsin substrate, BANA. Plaque was collected for BANA analysis from the surgical site prior to surgery. A curette was used for plaque collection from the site. After incubation for $5 \mathrm{~min}$. at $35^{\circ} \mathrm{C}$ with Evan's black dye solution, naphthylamine was released as a result of the presence of any one of the "redcomplex" pathogens and formed a permanent blue-black colour. The test was read as positive or negative based on the relative intensity of the blue colour.

\section{Tissue processing and immunohistochemistry}

Immediately following procurement, the tissue samples were stored in vials containing $4 \%$ vol formaldehyde and phosphatase inhibitor cocktail (PhosSTOP, Roche Diagnostic, Germany). After 24-36 h they were transferred 
into $70 \%$ ethanol, embedded in paraffin and then sections were prepared. An attempt was made to cut the sections in such a manner so that every sample would have both the epithelium and underlying connective tissue whenever possible. Immunohistochemical staining for p38, phospho-p38, ERK, phospho-ERK, JNK and phospho-JNK was performed. Deparaffinized ethanol-dehydrated tissue sections were placed in a pressure chamber (Biocare Medical, Concord, CA, USA) for $15 \mathrm{~min}$. in an antigen retrieval buffer (DAKO, Glostrup, Denmark) and allowed to cool to room temperature. Primary antibodies for p38, phospho-p38, ERK, phospho-ERK, JNK and phospho-JNK (Cell Signaling, Beverly, MA, USA; R\&D Systems, Minneapolis, MN, USA and Abcam, Cambridge, MA, USA) were used to evaluate the extent of expression, detected using Vectastain Elite ABC reagent and Nova Red (Vector Laboratories, Burlingame, CA, USA) per manufacturer's instructions. Control sections were incubated as well with pre-immune serum or control IgG. An independent oral pathologist (NJD), blinded to the periodontal status of the subjects, completed the immunohistochemical staining scoring analysis of the inflammatory infiltrate in the connective tissue as previously described (Kirkwood et al. 2007b, Mitra et al. 2008). Due to high background staining, the epithelium was not scored. The examiner first went through all the slides for a particular stain and selected slides that represented each of the four levels of intensity. All slides were scored using reference slides as the standard. All slides were assigned a score of $0,1,2$ or 3 , where " 0 = none", " 1 = low", " 2 = medium/ moderate" and "3 = high/severe" positive stain.

\section{Inflammation intensity scoring}

Serial sections from periodontal tissue samples were sectioned for standard haematoxylin and eosin (H\&E) staining to correlate with immunohistochemical (IHC) staining. Specimens were assessed and read for the degree of inflammatory infiltrate by a blinded oral pathologist (NJD). Infiltrate intensity was scored as follows: none (less than mild), mild, moderate or severe.

\section{Statistical analysis}

All statistical analyses were performed using $\mathrm{R}$ version 2.12.1 (available at: http://cran.r-project.org/bin/ windows/base/old/2.12.1/). The raw immunohistochemical staining scores and periodontal outcomes of GI and plaque index (PI) are presented in table format. PD measurements associated with the tooth from which the tissue sample was taken were summarized using the periodontal inflammatory burden index, PIBI, defined as the number of PD measurements of 4 or $5 \mathrm{~mm}$ plus twice the number of PD measurements $\geq 6 \mathrm{~mm}$ (Lindy et al. 2008, Leppilahti et al. 2011). We defined a summary
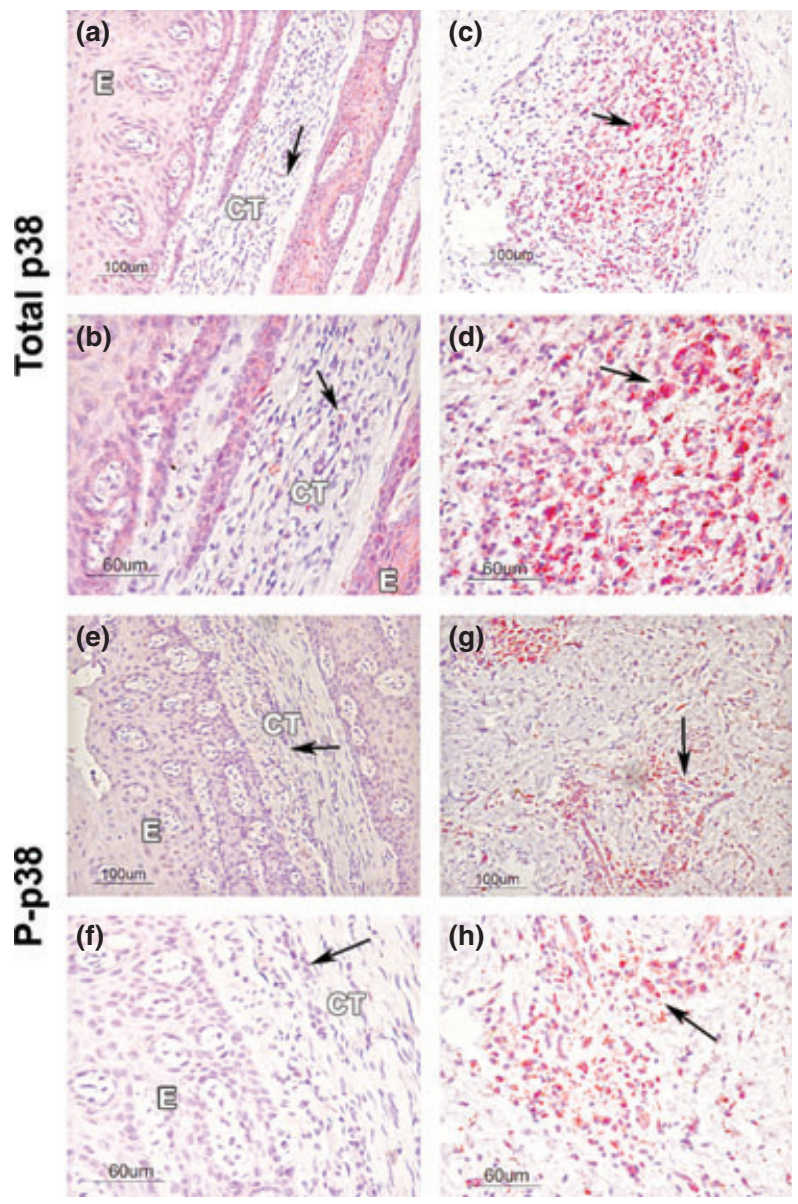

Fig. 1. Total (a-d) and Phospho (e-h) p38 mitogen activating protein kinases immunohistochemistry scoring. The left column (a, b, e and f) illustrates a " 0 " score (no staining) of immunostained tissue samples. The right column (c, d, g and h) illustrates a high/severe level of " 3 " immunostained tissue samples. "E" refers to epithelium and "CT" refers to connective tissue. Arrows indicate representative areas of the inflammatory infiltrate scored. 
obtained by back transforming normality-based confidence intervals for the Fisher transformation (Fisher 1915) of the estimated correlation. Because Fisher's transformation is non-linear, this back transformation of the symmetrical normality-based confidence interval results in an asymmetric confidence interval for the raw (untransformed) correlation.

\section{Results}

Subsets of MAPK expression correlates with inflammation and microbiological assessments

Twenty-one samples of periodontal tissues were collected from healthy subjects and chronic periodontitis patients. In the healthy group, nine samples of ten $(90 \%)$ were graded as "none - no inflammatory infiltrate observed" with scattered focally present lymphocytes and occasionally plasma cells. One sample was graded as mild and in that case focally present lymphocytes, plasma cells and macrophages were observed. The diseased group consisted of no samples in the "none" category, four $(36 \%)$ in the "mild", six $(55 \%)$ in the "moderate" and one $(9 \%)$ in the "severe". The diseased group exhibited significantly more inflammatory infiltrate, and lymphocytes, plasma cells and macrophages were present in almost all the samples. Occasionally, bacterial colonies were observed and interpreted as dental calculus as well as extravasated erythrocytes. IHC staining was performed on tissues following extensive optimization for each of the following antibodies: total p38, JNK and ERK along with phosphorylated (active) p38, JNK and ERK. Representative examples of total and phospho-p38 (Fig. 1), total and phospho-JNK (Fig. 2) and total and phospho-ERK (Fig. 3) are presented.

The intensity of the IHC scoring and the intensity of the inflammatory infiltrate are both positively correlated with the clinical parameters (Tables 1,2). BANA scores showed a striking correlation with clinical disease parameters and IHC scoring ( $p$-values $\leq 0.02$ for $\mathrm{p} 38$, phosphop38, ERK and JNK; BANA scores were not significantly correlated with phospho-ERK and phospho-JNK).
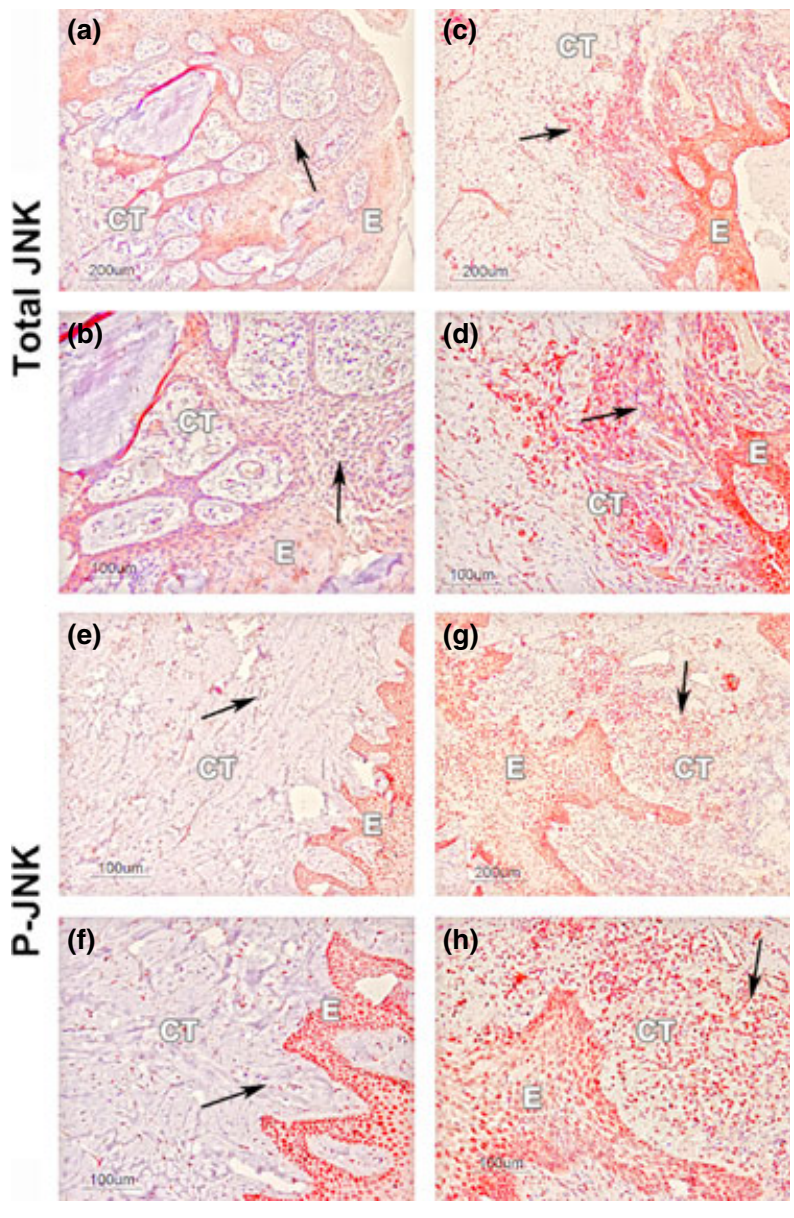

Fig. 2. Total (a-d) and Phospho (e-h) c-Jun N-terminal activated kinases mitogen activating protein kinases immunohistochemistry scoring. The left column ( $a, b$, e and $f$ ) illustrates a " 0 " score (no staining) of immunostained tissue samples. The right column (c, d, g and h) illustrates a high/severe level of " 3 " immunostained tissue samples. "E" refers to epithelium and "CT" refers to connective tissue. Arrows indicate representative areas of the inflammatory infiltrate scored.

Figure 4 depicts the estimated correlations between the clinical parameters and IHC scoring together with $95 \%$ confidence intervals computed as described in the Methods section. The confidence intervals reinforce the pattern of association between the clinical parameters and MAPK expression. Specifically, the clinical parameters are consistently, significantly, and positively associated with the IHC scoring for p38, phospho-p38, ERK and JNK (with the sole exception that the confidence interval for the association between JNK and the summary index PIBI just covers zero; $p$-value $=0.06$ from Table 2), and lack statistically significant correlation with phospho-ERK and phospho-JNK. Thus, MAPK expression, inflammatory infiltrate and
BANA exhibit similar patterns of association with the clinical parameters.

\section{Discussion}

Pro-inflammatory cytokines have long been identified as major pathogenic mediators involved in the pathobiology of both rheumatoid arthritis periodontitis, inducing and propagating a chronic inflammatory process leading towards tissue and bone destruction (Kirkwood et al. 2007b). In humans, MAPK activation has been evaluated in tissues from rheumatoid arthritis patients which has been well characterized as a chronic inflammatory disease where inflammatory, immunological and physical stimuli ultimately leading to tissue destruction (Zvaifler 

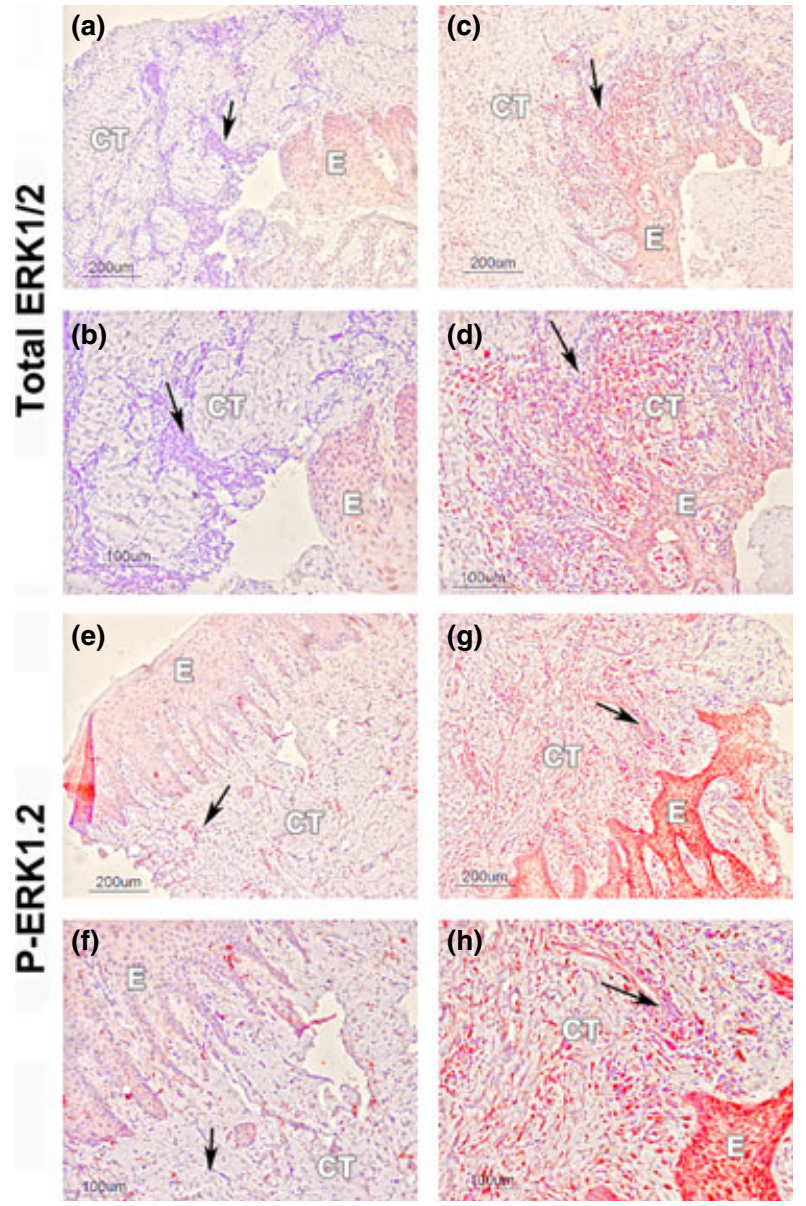

Fig. 3. Total (a-d) and Phospho (e-h) extracellular activated kinases mitogen activating protein kinases immunohistochemistry scoring. The left column ( $a, b$, e and $f$ ) illustrates a " 0 " score (no staining) of immunostained tissue samples. The right column (c, d, g and h) illustrates a high/severe level of " 3 " immunostained tissue samples. "E" refers to epithelium and "CT" refers to connective tissue. Arrows indicate representative areas of the inflammatory infiltrate scored.

1995a, Zvaifler 1995b, Firestein 1999). MAPK signalling is vital for synthesis and amplification of proinflammatory mediators and MMPs by synovial cells, chemoattraction of mononuclear cells, and angiogenesis of endothelial cells, as well as in synovial cell apoptosis (Schett et al. 2000 ), and $\mathrm{p} 38 \alpha$ and $\mathrm{p} 38 \beta$ seemed to be predominantly activated in the inflamed tissue (Korb et al. 2006). Given the similarities in cytokine networks between RA and periodontitis, we sought to understand if some of the major MAPK signalling pathways are operative during periodontal disease progression.

This study evaluated and compared the expression and activation of p38, ERK and JNK MAPKs in gingival tissues from normal healthy subjects and subjects with chronic periodontitis. Activation of total p38, ERK and JNK in diseased tissues was evident but an even more significant finding was the observation of more intense immunoreactivity for phosphorylated p38 in diseased tissues compared to controls (Tables 1, 2 and Fig. 4) suggesting increased phosphorylation of p38 in human chronic periodontitis. These findings are in agreement with results from studies that demonstrated activation of p38, ERK and JNK in samples from patients with rheumatoid arthritis, and that p38, specifically isoforms $\alpha$ and $\beta$ dominate in chronic inflammation (Schett et al. 2000, Korb et al. 2006). Although initial aetiology is different between periodontitis and rheumatoid arthritis, the signalling pathways used in response to bacte-
Table 1. Rank correlations for the inflammatory infiltrate scores and BANA test with MAPK intensities and clinical indices

\begin{tabular}{|c|c|c|c|c|}
\hline \multirow[t]{2}{*}{ MAPK } & \multicolumn{2}{|c|}{ Infiltrate } & \multicolumn{2}{|c|}{ BANA } \\
\hline & $\begin{array}{l}\text { Rank } \\
\text { corr. }\end{array}$ & $p$-value & $\begin{array}{l}\text { Rank } \\
\text { corr. }\end{array}$ & $p$-value \\
\hline p38 & 0.60 & 0.004 & 0.65 & 0.001 \\
\hline p.p38 & 0.53 & 0.012 & 0.50 & 0.020 \\
\hline ERK & 0.62 & 0.003 & 0.62 & 0.003 \\
\hline p.ERK & 0.43 & 0.051 & 0.39 & 0.084 \\
\hline JNK & 0.58 & 0.006 & 0.56 & 0.008 \\
\hline p.JNK & 0.29 & 0.196 & 0.19 & 0.411 \\
\hline \multicolumn{5}{|c|}{ Clinical indices } \\
\hline PI & 0.96 & $1 \mathrm{e}-11$ & 0.88 & $2 \mathrm{e}-07$ \\
\hline GI & 0.91 & $8 \mathrm{e}-09$ & 0.96 & $7 \mathrm{e}-12$ \\
\hline PIBI & 0.87 & $4 \mathrm{e}-07$ & 0.90 & $4 \mathrm{e}-08$ \\
\hline CALI & 0.84 & $2 \mathrm{e}-06$ & 0.89 & $5 e-08$ \\
\hline
\end{tabular}

BANA, benzoyl-DL-arginine-naphthylamide; CALI, clinical attachment level index; ERK, extracellular activated kinases; GI, Gingival Index; JNK, c-Jun N-terminal activated kinases; MAPK, mitogen activating protein kinases PIBI, periodontal inflammatory burden index.

rial challenge or proinflammatory cytokines are comparable. In fact, many of the same cytokines (including TNF- $\alpha$, IL- $1 \beta$ and IL-6) are associated with progression in both diseases. Data from periodontal disease pre-clinical models using a p38 $\alpha$ specific inhibitor suggest that at least p38 $\alpha$ may be a major p38 isoform involved in inflammatory signalling in chronic periodontitis (Kirkwood et al. 2007b).

Although this study is the first to address MAPK activation in human periodontitis, previous studies addressed NF- $\kappa \mathrm{B} \quad(\mathrm{p} 50 / \mathrm{p} 65)$ and inhibitor of kappa $\mathrm{B}(\mathrm{I} \kappa \mathrm{B})$ expression in chronic periodontitis patients where activation of NF- $\kappa \mathrm{B}(\mathrm{p} 50 / \mathrm{p} 65)$ was more significantly expressed in diseased periodontal tissues (Ambili et al. 2005). NF- $\kappa$ B and MAPK activation have also been shown to be instrumental in progression of inflammatory diseases including rheumatoid arthritis, cardiovascular disease (Cook et al. 1999, Schett et al. 2000), Alzheimer's disease (Culbert et al. 2006) and cancer progression (Hanahan \& Weinberg 2000).

Clinical data and histological grading of periodontal lesions presented agrees with the historical literature in clinical periodontics (Page \& Schroeder 1976) providing assurance that correlative data presented here relative to MAPK expression and 
Table 2. Rank correlations between MAPK scoring and clinical indices

\begin{tabular}{|c|c|c|c|c|c|c|c|c|}
\hline & \multicolumn{2}{|c|}{ PI } & \multicolumn{2}{|c|}{ GI } & \multicolumn{2}{|c|}{ PIBI } & \multicolumn{2}{|c|}{ CALI } \\
\hline & $\begin{array}{l}\text { Rank } \\
\text { corr. }\end{array}$ & $p$-value & $\begin{array}{l}\text { Rank } \\
\text { corr. }\end{array}$ & $p$-value & $\begin{array}{l}\text { Rank } \\
\text { corr. }\end{array}$ & $p$-value & $\begin{array}{l}\text { Rank } \\
\text { corr. }\end{array}$ & $p$-value \\
\hline p38 & 0.57 & 0.007 & 0.68 & 0.001 & 0.49 & 0.023 & 0.62 & 0.003 \\
\hline p.p38 & 0.44 & 0.047 & 0.47 & 0.034 & 0.45 & 0.039 & 0.56 & 0.009 \\
\hline ERK & 0.52 & 0.015 & 0.58 & 0.005 & 0.56 & 0.008 & 0.58 & 0.006 \\
\hline p.ERK & 0.35 & 0.116 & 0.40 & 0.071 & 0.25 & 0.269 & 0.34 & 0.135 \\
\hline JNK & 0.45 & 0.042 & 0.55 & 0.009 & 0.42 & 0.060 & 0.59 & 0.005 \\
\hline p.JNK & 0.31 & 0.165 & 0.15 & 0.530 & 0.17 & 0.450 & 0.20 & 0.386 \\
\hline
\end{tabular}

CALI, clinical attachment level index; ERK, extracellular activated kinases; GI, Gingival Index; JNK, c-Jun N-terminal activated kinases; MAPK, mitogen activating protein kinases; PIBI, periodontal inflammatory burden index.

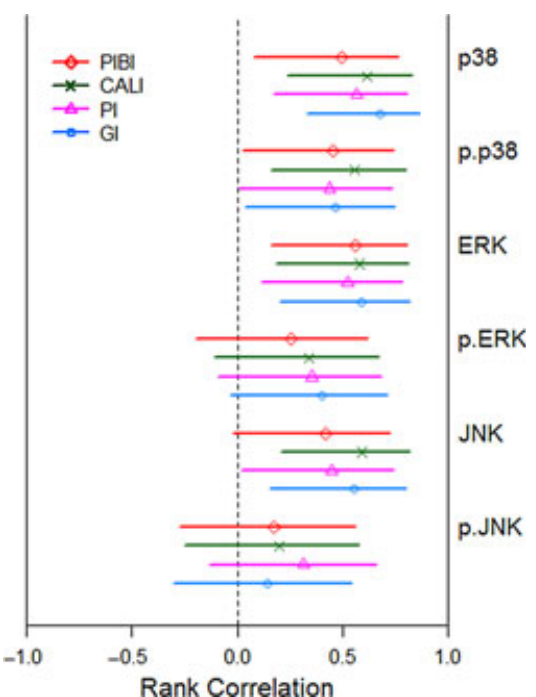

Fig 4. Association between the clinical periodontal indices and expression of mitogen activating protein kinases. The Spearman rank correlation and $95 \%$ confidence interval are shown. PI, plaque index; GI, gingival index; PIBI, periodontal inflammatory burden index; CALI, clinical attachment level index.

activation is compelling. Intensity scoring from immunostained tissues presented in Tables 1, 2 along with graphical representation of these data in Fig. 4 was correlated with clinical periodontal parameters. Rank correlations with clinical indices were significantly positive for total p38, phospho-p38 and total ERK expression levels, along with total JNK levels. Phospho-JNK and phospho-ERK showed no significant positive correlation with clinical parameters of disease or BANA positivity. Since there was an increase in the inflammatory infiltrate in the diseased versus healthy sites, there was an increase in the number of nuclei within these sites. Thus, the total amount of p38, JNK and ERK expression maybe positively correlated with clinical indices since there were more cells within the periodontal lesions. The lack of correlative data with p-JNK and p-ERK suggests that although there is more total JNK and ERK, these MAPKs are not significantly active during periodontal disease progression in humans. One of the potential shortcomings of these data resides in the inability to express the phosphorylated to non-phosphorylated MAPKs ratios due to lack of sufficient amount of quality serial samples. However, it should be noted again, that the connective tissue was only scored and reported here since there was high background immunostaining with the epithelium.

It is important to note that diseased samples evaluated for inflammation, BANA and MAPK expression in this study all had initial periodontal therapy including scaling and root planing prior to tissue procurement. Thus, the levels of inflammation observed are most likely lower then what would be expected in an untreated periodontal lesion, suggesting that the significance of the data presented here is highly significant. However, although BANA data correlated with periodontal disease status, these studies cannot conclude that all periodontal pathogens affect MAPK signalling pathways to the same extent or manner.

With emerging treatment modalities used to manage periodontitis that have been designed to modulate the host response as it has been recognized that the host response, not the bacterial infection, is primarily responsible for the connective tissue destruction in chronic periodontitis. This study confirms the findings from studies in small animal models demonstrating increased activation of MAPKs in periodontal disease progression (Kirkwood et al. 2007b), indicating that both increased MAPK expression and activation occurs with increased severity of periodontal disease in human tissues. Moreover, this is the first time that the levels of activation of MAPKs have been correlated with clinical parameters as well as BANA microbiological test results. These findings suggest that MAPK signalling plays a significant part of the ontogeny of inflammation in periodontal disease ultimately resulting in alveolar bone loss.

\section{Acknowledgements}

The authors acknowledge the support of the Histology Core Facility at the University of Michigan School of Dentistry, the MUSC Center for Oral Health Research, and Ms. Bethany Herbert for her assistance with digital imaging.

\section{References}

Ambili, R., Santhi, W. S., Janam, P., Nandakumar, K. \& Pillai, M. R. (2005) Expression of activated transcription factor nuclear factorkappaB in periodontally diseased tissues. Journal of Periodontology 76, 1148-1153.

Assuma, R., Oates, T., Cochran, D., Amar, S. \& Graves, D. T. (1998) IL-1 and TNF antagonists inhibit the inflammatory response and bone loss in experimental periodontitis. The Journal of Immunology 160, 403-409.

Beklen, A., Hukkanen, M., Richardson, R. \& Konttinen, Y. T. (2008) Immunohistochemical localization of Toll-like receptors 1-10 in periodontitis. Oral Microbiology and Immunology 23 , 425-431.

Cook, S. A., Sugden, P. H. \& Clerk, A. (1999) Activation of c-Jun N-terminal kinases and p38-mitogen-activated protein kinases in human heart failure secondary to ischaemic heart disease. Journal of Molecular and Cellular Cardiology 31, 1429-1434.

Culbert, A. A., Skaper, S. D., Howlett, D. R., Evans, N. A., Facci, L., Soden, P. E., Seymour, Z. M., Guillot, F., Gaestel, M. \& Richardson, J. C. (2006) MAPK-activated protein kinase 2 deficiency in microglia inhibits pro-inflammatory mediator release and resultant neurotoxicity. Relevance to neuroinflammation in a transgenic mouse model of Alzheimer disease. The Journal of Biological Chemistry 281, 23658-23667.

Ejeil, A. L., Gaultier, F., Igondjo-Tchen, S., Senni, K., Pellat, B., Godeau, G. \& Gogly, B. (2003) Are cytokines linked to collagen breakdown during periodontal disease progression? Journal of Periodontology 74, 196-201. 
Firestein, G. S. (1999) Starving the synovium: angiogenesis and inflammation in rheumatoid arthritis. The Journal of Clinical Investigation 103, 3-4.

Fisher, R. A. (1915) Frequency distribution of the values of the correlation coefficient in samples of an indefinitely large population. Biometrika (Biometrika Trust) 10, 507-521.

Gamonal, J., Acevedo, A., Bascones, A., Jorge, O. \& Silva, A. (2000) Levels of interleukin-1 beta, -8 , and -10 and RANTES in gingival crevicular fluid and cell populations in adult periodontitis patients and the effect of periodontal treatment. Journal of Periodontology 71, 1535-1545.

Geivelis, M., Turner, D. W., Pederson, E. D. \& Lamberts, B. L. (1993) Measurements of interleukin-6 in gingival crevicular fluid from adults with destructive periodontal disease. Journal of Periodontology 64, 980-983.

Gorska, R., Gregorek, H., Kowalski, J., LaskusPerendyk, A., Syczewska, M. \& Madalinski, K. (2003) Relationship between clinical parameters and cytokine profiles in inflamed gingival tissue and serum samples from patients with chronic periodontitis. Journal of Clinical Periodontology 30, 1046-1052.

Graves, D. T. (1999) The potential role of chemokines and inflammatory cytokines in periodontal disease progression. Clinical Infectious Diseases 28, 482-490.

Hanahan, D. \& Weinberg, R. A. (2000) The hallmarks of cancer. Cell 100, 57-70.

Jiang, Y., Mehta, C. K., Hsu, T. Y. \& Alsulaimani, F. F. (2002) Bacteria induce osteoclastogenesis via an osteoblast-independent pathway. Infection and Immunity 70, 3143-3148.

Kikuchi, T., Yoshikai, Y., Miyoshi, J., Katsuki, M., Musikacharoen, T., Mitani, A., Tanaka, S., Noguchi, T. \& Matsuguchi, T. (2003) Cot/ $\mathrm{Tpl} 2$ is essential for RANKL induction by lipid A in osteoblasts. Journal of Dental Research 82, 546-550.

Kirkwood, K. L., Cirelli, J. A., Rogers, J. E. \& Giannobile, W. V. (2007a) Novel host response therapeutic approaches to treat periodontal diseases. Periodontology 2000 43, 294-315.

Kirkwood, K. L., Li, F., Rogers, J. E., Otremba, J., Coatney, D. D., Kreider, J. M., D'Silva, N. J., Chakravarty, S., Dugar, S., Higgins, L. S., Protter, A. A. \& Medicherla, S. (2007b) A p38alpha selective mitogen-activated protein kinase inhibitor prevents periodontal bone loss. The Journal of Pharmacology and Experimental Therapeutics 320, 56-63.

Kirkwood, K. L. \& Rossa, C. Jr (2009) The potential of p38 MAPK inhibitors to modulate periodontal infections. Current Drug Metabolism 10, 55-67.

Kirschning, C. J. \& Bauer, S. (2001) Toll-like receptors: cellular signal transducers for exogenous molecular patterns causing immune responses. International Journal of Medical Microbiology 291, 251-260.

Kirschning, C. J., Wesche, H., Merrill Ayres, T. \& Rothe, M. (1998) Human toll-like receptor 2 confers responsiveness to bacterial lipopolysaccharide. Journal of Experimental Medicine 188, 2091-2097.

Korb, A., Tohidast-Akrad, M., Cetin, E. Axmann, R., Smolen, J. \& Schett, G. (2006) Differential tissue expression and activation of p38 MAPK alpha, beta, gamma, and delta isoforms in rheumatoid arthritis. Arthritis and Rheumatism 54, 2745-2756.

Lee, H. J., Kang, I. K., Chung, C. P. \& Choi, S. M. (1995) The subgingival microflora and gingival crevicular fluid cytokines in refractory periodontitis. Journal of Clinical Periodontology 22, 885-890.

Leppilahti, J. M., Ahonen, M. M., Hernandez, M., Munjal, S., Netuschil, L., Uitto, V. J., Sorsa, T. \& Mantyla, P. (2011) Oral rinse MMP-8 point-of-care immuno test identifies patients with strong periodontal inflammatory burden. Oral Diseases $\mathbf{1 7}$ 115-122.

Li, Q., Valerio, M. S. \& Kirkwood, K. L. (2012) MAPK usage in periodontal disease progression. Journal of Signal Transduction 2012, 308943.

Li, Q., Yu, H., Zinna, R., Martin, K., Herbert, B., Liu, A., Rossa, C. . Jr \& Kirkwood, K. L. (2011) Silencing mitogen-activated protein kinase-activated protein kinase-2 arrests inflammatory bone loss. The Journal of Pharmacology and Experimental Therapeutics 336, 633-642.

Lindy, O., Suomalainen, K., Makela, M. \& Lindy, S. (2008) Statin use is associated with fewer periodontal lesions: A retrospective study. BMC Oral Health 8, 16.

Loe, H. (1967) The gingival index, the plaque index and the retention index systems. Journal of Periodontology 38 (Suppl) 610-616.

Loesche, W. J., Bretz, W. A., Kerschensteiner, D., Stoll, J., Socransky, S. S., Hujoel, P. \& Lopatin, D. E. (1990) Development of a diagnostic test for anaerobic periodontal infections based on plaque hydrolysis of benzoyl-DL-argininenaphthylamide. Journal of Clinical Microbiology 28, 1551-1559.

Mitra, R. S., Goto, M., Lee, J. S., Maldonado, D., Taylor, J. M., Pan, Q., Carey, T. E., Bradford, C. R., Prince, M. E., Cordell, K. G., Kirkwood, K. L. \& D’Silva, N. J. (2008) Rap1GAP promotes invasion via induction of matrix metalloproteinase 9 secretion, which is associated with poor survival in low $\mathrm{N}$-stage squamous cell carcinoma. Cancer Research $\mathbf{6 8}$, 3959-3969.

Mori, Y., Yoshimura, A., Ukai, T., Lien, E., Espevik, T. \& Hara, Y. (2003) Immunohistochemical localization of Toll-like receptors 2 and 4 in gingival tissue from patients with periodontitis. Oral Microbiology and Immunology 18, 54-58.

Nakashima, T., Kobayashi, Y., Yamasaki, S., Kawakami, A., Eguchi, K., Sasaki, H. \& Sakai, H. (2000) Protein expression and functional difference of membrane-bound and soluble receptor activator of NF-kappaB ligand: modulation of the expression by osteotropic factors and cytokines. Biochemical and Biophysical Research Communications 275, 768-775.

Page, R. C. \& Schroeder, H. E. (1976) Pathogenesis of inflammatory periodontal disease. A summary of current work. Laboratory Investigation 34, 235-249.

Palanisamy, V., Jakymiw, A., Van Tubergen, E. A., D'Silva, N. J. \& Kirkwood, K. L. (2012) Control of Cytokine mRNA Expression by RNA-binding Proteins and microRNAs. Journal of Dental Research 91, 651-658.

Reddi, K., Wilson, M., Nair, S., Poole, S. \& Henderson, B. (1996) Comparison of the pro-inflammatory cytokine-stimulating activity of the surface-associated proteins of periodontopathic bacteria. Journal of Periodontal Research 31, 120-130.

Rogers, J. E., Li, F., Coatney, D. D., Otremba, J., Kriegl, J. M., Protter, T. A., Higgins, L. S., Medicherla, S. \& Kirkwood, K. L. (2007) A p38 mitogen-activated protein kinase inhibitor arrests active alveolar bone loss in a rat periodontitis model. Journal of Periodontology 78, 1992-1998.

Schett, G., Tohidast-Akrad, M., Smolen, J. S., Schmid, B. J., Steiner, C. W., Bitzan, P., Zenz, P., Redlich, K., Xu, Q. \& Steiner, G. (2000) Activation, differential localization, and regulation of the stress-activated protein kinases, extracellular signal-regulated kinase, c-JUN Nterminal kinase, and p38 mitogen-activated protein kinase, in synovial tissue and cells in rheumatoid arthritis. Arthritis and Rheumatism 43, 2501-2512.

Stashenko, P., Jandinski, J. J., Fujiyoshi, P. Rynar, J. \& Socransky, S. S. (1991) Tissue levels of bone resorptive cytokines in periodontal disease. Journal of Periodontology 62, 504-509.

Yang, H., Young, D. W., Gusovsky, F. \& Chow, J. C. (2000) Cellular events mediated by lipopolysaccharide-stimulated toll-like receptor 4 . MD-2 is required for activation of mitogenactivated protein kinases and Elk-1. Journal of Biological Chemistry 275, 20861-20866.

Zvaifler, N. J. (1995a) Macrophages and the synovial lining. Scandinavian Journal of Rheumatology 101, 67-75.

Zvaifler, N. J. (1995b) Rheumatoid arthritis. The multiple pathways to chronic synovitis. Laboratory Investigation 73, 307-310.

\section{Address:}

Keith Kirkwood

Department of Craniofacial

Biology and Center for Oral Health

Research, Medical University of South

Carolina, 173 Ashley Ave. 254BS

Charleston, 29425 SC

USA

E-mail: klkirk@musc.edu 


\section{Clinical Relevance}

Scientific rationale for the study: This study provides basic biochemical data regarding the expression of mitogen activating protein kinase (MAPK) in diseased and healthy human periodontal tissues. MAPKs are activated via phosphorylation in response to bacterial exposure and are critical mediators of chronic inflammation through the production of pro-inflammatory cytokines. Previous data from the project laboratory have shown that blocking specific MAPK reduced experimental periodontitis.

Principal findings: This study shows that certain MAPK are more highly activated in clinically diseased versus healthy tissue counterparts.

Practical implications: These data may have clinically important information since MAPK can be targeted for therapeutic benefit. 